\title{
Methemoglobinemia Caused by an Inert Ingredient after Intentional Ingestion of Pesticide
}

Ru Bi Jeong, M.D., Chang Hwan Sohn, M.D., Dong Woo Seo, M.D., Won Young Kim, M.D., Seung Mok Ryoo, M.D., Bum Jin Oh, M.D., and Kyoung Soo Lim, M.D.

Asan Medical Center, University of Ulsan College of Medicine, Seoul, Korea

We report two cases of toxic methemoglobinemia caused by an inert ingredient in pesticide product after intentional ingestion of pesticide. First, 51-year-old male visited to the emergency department (ED) after the ingestion of pesticide in a suicide attempt. Initial methemoglobin (MetHb) level was $25.6 \%$. We did not know the cause of methemoglobinemia at that time. Second, 56 -year-old female visited to the ED after the ingestion of the same pesticide in a suicide attempt. MetHb level after 30 minutes was $16.1 \%$. The patients were treated with methylene blue. We contacted to the Korean Rural Development Administration and estimated that magnesium nitrate was more likely to cause methemoglobinemia. This report highlights the importance of considering the possibility of methemoglobinemia caused by inert ingredient in pesticide and early antidotal therapy.

Key Words: methemoglobinemia; pesticides.

Methemoglobinemia occurs when hemoglobin is oxidized to form methemoglobin (MetHb), rendering it incapable of oxygen transport and if severe, it leads to tissue hypoxia.[1] Most commonly, acquired methemoglobinemia can arise after exposure to an exogenous oxidizing agent and a variety of compounds are capable of inducing methemoglobinemia.[1] Cases of methemoglobinemia caused by indoxacarb, the active ingredient in some pesticides, have been reported.[2-5] However, to date, no cases of methemoglobinemia caused by an inert ingredient in pesticide have been reported. We report herein two cases of toxic methemoglobinemia caused by an inert ingredient in pesticide

Received on February 27, 2014 Revised on June 24, 2014

Accepted on August 28, 2014

Correspondence to: Chang Hwan Sohn, Asan Medical Center, University of Ulsan College of Medicine, 88 Olympic-ro, 43-gil, Songpa-gu, Seoul 138-736, Korea

Tel: +82-2-3010-3350, Fax: +82-2-3010-3360

E-mail: schwan97@gmail.com

ORCID: http://orcid.org/0000-0001-9747-0196

* No potential conflict of interest relevant to this article was reported. product after intentional ingestion of pesticide.

\section{Case Report}

\section{Case 1}

A previously healthy 51-year-old male visited to the nearest emergency department (ED) with a history of ingestion of three mouthfuls of pesticide (Sonanae Inc., Korea) containing 21.3\% of mefenacet (CAS registry number 73250-68-7) and 1.3\% of cyclosulfamuron (CAS registry number 136849-15-5) as active ingredients in a suicide attempt. He had no past medical problems. He received gastric lavage with $10 \mathrm{~L}$ of normal saline and $50 \mathrm{~g}$ of activated charcoal. During close observation, dyspnea and cyanosis developed and then MetHb concentration was measured about 4 hours later after ingestion of pesticide. Initial MetHb level was 25.6\%. He was transferred to our ED for antidote therapy. On arrival to the ED, he was drowsy mentality. His blood pressure was 134/95 $\mathrm{mmHg}$, pulse rate was 84 beats/minute, respiratory rate was 20 breaths/minute, and oxygen saturation in pulse oxymeter was $91 \%$. He had dyspnea and lip 


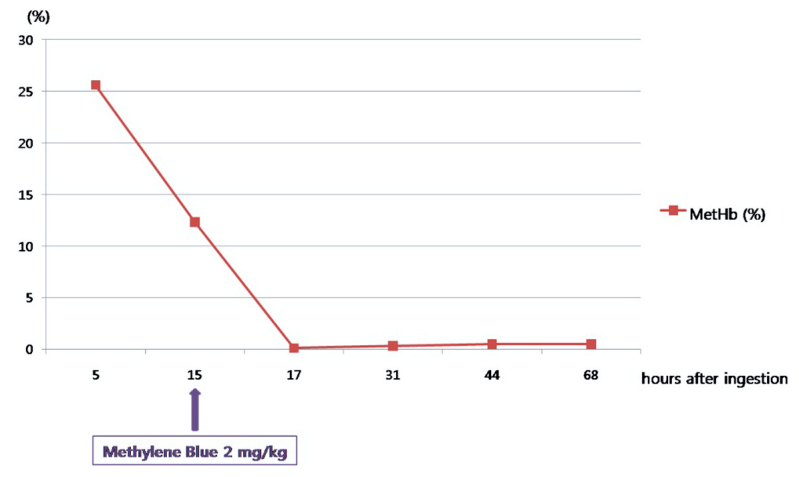

Fig. 1. Methemoglobin levels in case 1.

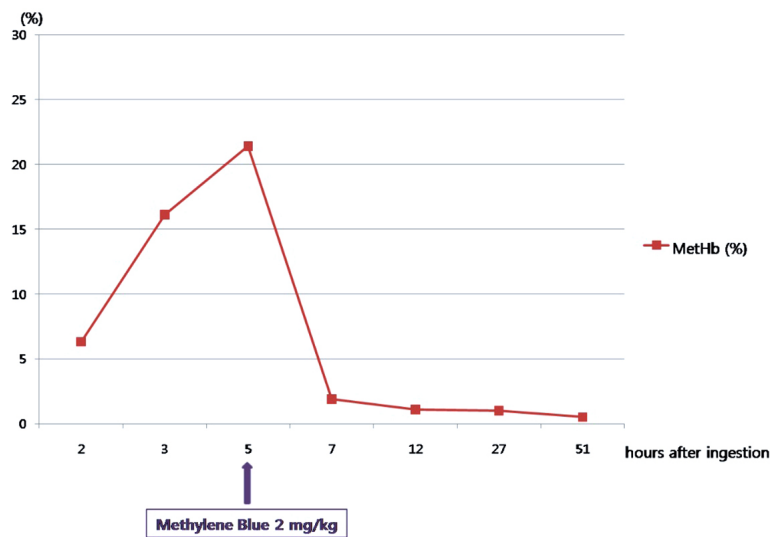

Fig. 2. Methemoglobin levels in case 2.

cyanosis. Initial MetHb in our ED was $12.3 \%$. But because she constantly complained of shortness of breath, we decided to use methylene blue. He received a single dose infusion of methylene blue ( $2 \mathrm{mg} / \mathrm{kg}$ body weight intravenously) over 10 minutes about 16 hours later after ingestion of pesticide. And then his symptoms were relieved. Methemoglobin level on follow up was $0.1 \%$ (Fig. 1). On psychiatric consultation, he was considered low risk for a suicide reattempt and close outpatient follow-up with psychiatry was decided. Three days after admission, he was discharged without any symptoms or signs. We did not know the cause of methemoglobinemia at that time.

\section{Case 2}

A previously healthy 56-year-old female visited to the nearest emergency department with a history of ingestion of two cups of pesticide (Sonanae Inc., Korea) containing 21.3\% of mefenacet (CAS registry number 73250-68-7) and $1.3 \%$ of cyclosulfamuron (CAS registry number 136849-15-5) as active ingredients in a suicide attempt. She had no past medical problems. She received gastric lavage and activated charcoal. During close observation, dyspnea developed and then $\mathrm{MetHb}$ concentration was measured about 100 minutes later after ingestion of pesticide. Initial MetHb level was 6.3\% and MetHb level on follow up was $16.1 \%$ after 30 minutes. She was transferred to our emergency department for antidote therapy. On arrival to the ED, he was drowsy mentality. Her blood pressure was $151 / 81 \mathrm{mmHg}$, pulse rate was 61 beats/minute, respiratory rate was 20 breaths/minute, and oxygen saturation in pulse oxymeter was $88 \%$. She had dyspnea, general weakness, and lip cyanosis. Initial MetHb in our ED was $21.4 \%$. She received a single dose infusion of methylene blue ( $2 \mathrm{mg} / \mathrm{kg}$ body weight intravenously) over 10 minutes about 5 hours later after ingestion of pesticide. And then her symptoms were relieved. MetHb on follow up was $1.1 \%$ (Fig. 2). On psychiatric consultation, she was considered moderate risk for a suicide reattempt and was encouraged to inpatient psychiatric hospitalization. But, three days after admission, she discharged against medical advice without any symptoms or signs. One week after the patient discharged, we contacted a pesticide expert in Korean Rural Development Administration and then could identify exact inert ingredients in the pesticide formulation. We estimated that of inert ingredients, magnesium nitrate was more likely to cause methemoglobinemia.

\section{Discussion}

In the present case, we identified that an inert ingredient in pesticide cause the life-threatening condition and methemoglobinemia was caused by magnesium nitrate used as a preservative, an inert ingredient in pesticide. To the best of our knowledge, this is the first report of methemoglobinemia caused by an inert ingredient in pesticide.

Pesticide products contain both active ingredients and inert ingredients. A significant proportion of typical pesticide formulations are inert ingredients. Inert ingredients serve a variety of function in pesticide formulations, acting as solvents, surfactants, or preservatives, among many other functions and despite their name, they may have biological activity of their own, they may be toxic to humans, and they may be chemically active. In some cases, inert ingredients can pose greater risks than active ingredients itself.[6]

Magnesium nitrate used as a preservative, an inert ingredient in some pesticides, has been known to cause methemoglobinemia.[7] Methemoglobinemia occurs when the iron moiety of hemoglobin is oxidized from the ferrous state $\left(\mathrm{Fe}^{2+}\right)$ to the ferric state $\left(\mathrm{Fe}^{3+}\right)$, forming methemoglobin (MetHb).[1] This change renders MetHb incapable of oxygen transport and shifts the oxygen dissociation curve leftward.[1,8] This reduces oxygen delivery, 
thereby increasing the risk of tissue hypoxia.[9] In general, symptoms of methemoglobinemia correlate with MetHb level in healthy patients. Cyanosis occurs at a MetHb level $>15 \%$ of total hemoglobin in nonanemic patients; anxiety, headache, and dizziness at a MetHb level $>20 \%$; fatigue, confusion, and tachypnea between $30 \%$ and $50 \%$; arrhythmias, acidosis, seizures, and even death at a MetHb level > 50\%.[8] In the present case, methemoglobinemia with peak MetHb level of $25.6 \%$ in case 1 and $21.4 \%$ in case 2 developed after intentional ingestion of the same pesticide containing magnesium nitrate as an inert ingredient. The mainstay of the treatment of acquired methemoglobinemia is identification and withdrawal of the offending agent.[9] Methylene blue is the treatment of choice in severe cases of methmeglobinemia.[10] Methylene blue should be given intravenously at a dose of 1 to $2 \mathrm{mg} / \mathrm{kg}$ for 5 minutes.[8] The maximum effect of methylene blue occurs quickly, at $30 \mathrm{mi}-$ nutes and if response is inadequate, additional dose of methylene blue may be given after 1 hour.[11] In the present case, both patients recovered from methemoglobinemia after a single dose infusion of methylene blue.

The authors initially could not identify inert ingredients in the product label and experienced that the process of identifying inert ingredients in the pesticide in South Korea caused substantial delay. There is a clear need for more public disclosure of the identity of all ingredients of pesticide products. Also, most of clinicians attending patients with pesticide poisoning will only consider the active ingredient in pesticide. However, as in the present case, an inert ingredient in pesticide should be considered and identified when treating patients with pesticide poisoning.

This would be a limitation of this case report that it is difficult to know the composition of magnesium sulfate in this pesticide. but magnesium nitrate is a very rare component of pesticides which is produced in Korea in recent years.

The present study highlights the importance of considering the possibility of toxicity caused by an inert ingredient in pesticide and in particular, methemoglobinemia in cases of exposure to an inert ingredient such as magnesium nitrate for its early rec- ognition and antidotal therapy.

\section{References}

1) Mansouri A, Lurie AA: Concise review: methemoglobinemia. Am J Hematol 1993; 42: 7-12.

2) Prasanna L, Rao SM, Singh V, Kujur R, Gowrishankar: Indoxacarb poisoning: an unusual presentation as methemoglobinemia. Indian J Crit Care Med 2008; 12: 198-200.

3) Wu YJ, Lin YL, Huang HY, Hsu BG: Methemoglobinemia induced by indoxacarb intoxication. Clin Toxicol (Phila) 2010; 48: 766-7.

4) Park JS, Kim H, Lee SW, Min JH: Successful treatment of methemoglobinemia and acute renal failure after indoxacarb poisoning. Clin Toxicol (Phila) 2011; 49: 744-6.

5) Jin K: Rhabdomyolysis, methemoglobinemia and acute kidney injury after indoxacarb poisoning. Clin Toxicol (Phila) 2012; 50: 227.

6) Cox C, Surgan M: Unidentified inert ingredients in pesticides: implications for human and environmental health. Environ Health Perspect 2006; 114: 1803-6.

7) Centre for Science of Athabasca University: Magnesium Nitrate Material Safety Data Sheet. Athabasca University [Lab Reagents Database] 2005 Oct [2005 Oct ]. Available from http://science.pc.athabascau.ca/reagentstud.nsf/0/f8e65 efce32b16c2872570920065283b/\$FILE/Magnesium\%20nitrate $\% 20$ hexahydrate $\% 20$ MSDS.pdf.

8) Wright RO, Lewander WJ, Woolf AD: Methemoglobinemia: etiology, pharmacology, and clinical management. Ann Emerg Med 1999; 34: 646-56.

9) Skold A, Cosco DL, Klein R: Methemoglobinemia: pathogenesis, diagnosis, and management. South Med J 2011; 104: 757-61.

10) Groeper K, Katcher K, Tobias JD: Anesthetic management of a patient with methemoglobinemia. South Med J 2003; 96 : 504-9.

11) Clifton J 2nd, Leikin JB: Methylene blue. Am J Ther 2003; 10: 289-91. 